A. Lorenzo: None declared, Nieves Martín: None declared, Carlos Rodriguez-Lozano: None declared

DOI: 10.1136/annrheumdis-2019-eular.928

Table

\begin{tabular}{lc}
\hline Age, mean (SD) & $73.4(10)$ \\
\hline BMI, mean & 27.4 \\
Fracture type, N (\%) & \\
Forearm & $71(18)$ \\
Hip & $132(34)$ \\
Humerus & $77(20)$ \\
Vertebra & $52(13)$ \\
Others & $49(13)$ \\
FRAX items, N (\%) & \\
Previous fracture & $51(13)$ \\
Hip fracture of parents & $36(9)$ \\
Smoking & $81(21)$ \\
Corticoids & $24(6)$ \\
Rheumatoid arthritis & $4(1)$ \\
Secondary osteoporosis & $17(4)$ \\
Alcohol & $68(18)$ \\
Densitometry (\%) & \\
Normal & 30 \\
Osteopenia & 43 \\
Osteoporosis & 26 \\
FRAX, mean (SD) & 26 \\
Major fracture & \\
Hip fracture & $8,4(5)$ \\
Treatment prescribed, (\%) & $4,3(4)$ \\
Bishoshonate or equivalent & \\
Refferal to primary care & 68 \\
Persistence at 12 months & 64 \\
\hline
\end{tabular}

\section{FRI0496 OSTEOPOROSIS IN PRIMARY CARE - ARE WE MISSING A TRICK?}

Sultana Parvin ${ }^{1}$, Manraj Barhey ${ }^{2}$, Talib Abubacker ${ }^{3}$, Muhammad Khurram Nisar ${ }^{1}$. ${ }^{1}$ Luton and Dunstable University Hospital, Rheumatology, Luton, United Kingdom; ${ }^{2}$ Woodland Avenue Practice, Primary car, Luton, United Kingdom; ${ }^{3}$ Bell House Medical centre, Primary care, Luton, United Kingdom

Background: Globally various incentive schemes have been employed in primary care to improve early diagnosis and management of several rheumatic conditions. In the UK, the Primary Care Quality and Outcomes Framework (QOF) rewards general practices for the provision of 'quality care' and helps to fund further improvements in the delivery of clinical care. Currently, there is one quality indicator in place for secondary prevention of osteoporosis. In order to help establish an integrated care pathway encompassing the whole patient journey between primary and secondary care, we undertook a detailed survey of two GP practices. Objectives: The aims of the exercise were to identify the utility of quality indicator and any gaps in the model of care for the high-risk osteoporosis patients.

Methods: An independent service evaluation tool was employed to interrogate the IT system used in the GP surgeries. All patients over the age of 65 were extracted from the database and FRAX analysis was undertaken. Those with medium to high FRAX score (i.e. ten-year risk of $>20 \%$ for major osteoporotic fracture and/or $>5 \%$ for hip fracture) were captured to explore whether they were offered further evaluation and bone-sparing therapy as necessary.

Results: Of 18,248 patients registered in the multi-cultural urban practices, 6796 were $>65$ years old. 793 had pre-defined moderate-high FRAX score. $300(37 \%)$ had a confirmed diagnosis of osteoporosis. Median age was 78 (range 65-103 years). 249 (83\%) were women. 88.5\% were White and remaining of other ethnicities.

$20 / 300(6.6 \%)$ had been coded to have ever sustained a fragility fracture. 178 (59.3\%) were prescribed bone-sparing therapy with five people taking it for over five years. $91 \%$ were prescribed oral therapy $(78 \%$ alendronic acid, $10 \%$ risedronic acid and $3 \%$ others) and remaining had parenteral therapies. Of the 27 patients not receiving any treatment, $11(30 \%)$ were incorrectly coded. The remainder's reasons for lack of treatment include intolerance, poor adherence and comorbidities.

Conclusion: This study highlights the inadequacy of quality indicators in the overall management of osteoporosis burden in primary care. It relies heavily on active identification process for high-risk individuals and correct coding of fragility fracture. However the vast majority of patients with moderate-high risk, based on case finding strategy advised by international bodies e.g. FRAX, remain hidden. Less than $10 \%$ of patients with confirmed osteoporosis fulfil the quality outcome in this survey. The QOF hence fails to reflect the nature of disease burden in the primary care thereby risking the management strategies skewed towards too small a cohort and missing the big picture. It is clear that quality indicators for osteoporosis need to be aligned to risk stratification model. This will allow better identification of at-risk individuals and improved care pathway for patients requiring bone active therapies.

Disclosure of Interests: Sultana Parvin: None declared, Manraj Barhey: None declared, Talib Abubacker: None declared, Muhammad Khurram Nisar Grant/research support from: Muhammad Nisar undertakes clinical trials and received support (including attendance at conferences, speaker fees and honoraria) from Roche, Chugai, MSD, Abbvie, Pfizer, BMS, Novartis, Celgene, Mallinckrodt, UCB and Lilly, Consultant for: Muhammad Nisar undertakes clinical trials and received support (including attendance at conferences, speaker fees and honoraria) from Roche, Chugai, MSD, Abbvie, Pfizer, BMS, Novartis, Celgene, Mallinckrodt, UCB and Lilly, Speakers bureau: Muhammad Nisar undertakes clinical trials and received support (including attendance at conferences, speaker fees and honoraria) from Roche, Chugai, MSD, Abbvie, Pfizer, BMS, Novartis, Celgene, Mallinckrodt, UCB and Lilly

DOI: 10.1136/annrheumdis-2019-eular.206

\section{FRI0497 TRABECULAR BONE SCORE AND MALNUTRITION IN A COHORT OF SYSTEMIC SCLEROSIS PATIENTS}

Sabrina Paolino ${ }^{1}$, Massimo Patanè ${ }^{1}$, Veronica Tomatis ${ }^{1}$, Andrea Casabella ${ }^{1}$, Carmen Pizzorni ${ }^{1}$, Carlotta Schenone ${ }^{1}$, Luca Carmisciano ${ }^{2}$, Alessio Signori ${ }^{2}$, Maurizio Cutolo'. 'IRCCS San Martino Polyclinic Hospital, Genoa, Italy, Research Laboratory and Academic Division of Clinical Rheumatology, Department of Internal Medicine, University of Genova, genoa, Italy; ${ }^{2}$ IRCCS San Martino Polyclinic Hospital, Genoa, Italy, 2Biostatistics Unit, Department of Health Sciences, University of Genoa, Genoa, Italy, genoa, Italy

Background: Systemic sclerosis (SSc) is a connective tissue disease characterized by initial microvascular damage, immune system activation and progressive fibrosis of the skin and internal organs.Gastrointestina (GI) involvement induce malnutrition due to gastroesophageal symptoms, Gl dismotility and malabsorption that are related to fibrosis of bowel wall and bacterial overgrowth(1).Therefore the disease is associated with secondary osteoporosis with a few studies evaluating the bone microarchitecture(2).

Objectives: To evaluate a relationship between malnutrition and bone microarchitecture detected by trabecular bone score (TBS) in SSc patients.

Methods: 38 patients( 6 male and 32 female) fulfilling ACR 2013 criteria for SSc underwent DXA to detect quantitative lumbar spine bone minera density and TBS. DXA also assess body composition with a software that provides the physician quantitative parameters, including free fat mass index (FFMI), that identifies the patient with malnutrition(values $<15$ $\mathrm{kg} / \mathrm{m} 2$ in women and $17 \mathrm{~kg} / \mathrm{m} 2$ in men), according to the ESPEN criteria (3).Body mass index was calculated for all SSc patients and every patient completed a diary reporting Gl symptoms possibly related to intestinal disbiosis.Fasting blood samples were obtained in order to analyse some biochemical parameters of malnutrition (total proteins $(\mathrm{g} / \mathrm{L})$, albumin $(\mathrm{g} /$ L), serum total cholesterol $(\mathrm{mg} / \mathrm{dl})$ and blood lymphocyte count $(\mathrm{N} / \mathrm{mm} 3)$. Continue variables were summarized as mean and standard deviation(SD) or median and inter quartile rang (IQR), discrete variables were summarized with count and percentage. Correlation was tested with Pearson or Spearman method. T-test was used to compare TBS between dichotomic groups Uni and multivariate linear regression models were used as well the Multiple R-squared variation was applied. The multivariate linear regression was performed with a stepwise approach to select the best model using highest AIC criteria.

Results: The mean age of patients was $64.2 \pm 11.3$ years with mean disease duration $19.2 \pm 7.6$ years. $36.8 \%$ of patients was found malnourished. The univariate analysis showed that only higher age of patients correlated to lower $\operatorname{TBS}(\mathrm{p}<0.001)$. The $\mathrm{R}$-squared of multivariate linear regression showed that about $45 \%$ of the TBS variations(TBSv) can be explained by the variation of the following variables(age, disease duration, lymphocyte count).Age explains about $25 \%$ of the TBSv.Older patients had lower TBS, with approximately 0.05 points of TBS loss every decade $(\mathrm{p}=$ 0.001).The presence of symptom possibly related to intestinal disbiosis, added to the model, might explains about $12 \%$ more of TBSv. Patients with symptom related to bacterial overgrowth had lower TBS respect to patients without(-0.08), regardless of other variables $(p=0.002)$.Disease duration, added to the model, further explains about $4 \%$ more of TBSv and suggest a trend between highest disease duration (regardless of other variables) and higher $\operatorname{TBS}(\mathrm{p}=0.103)$. Lymphocyte count added to 